\title{
An Innovative Cerclage Wire Passer
}

\author{
Hrishikesh Saodekar, Salphale Yogesh \\ Suyash Hospital, Rukmini Nagar, Amravati, India \\ Email:yosa@aol.in
}

How to cite this paper: Saodekar, H. and Yogesh, S. (2018) An Innovative Cerclage Wire Passer. Surgical Science, 9, 197-202. https://doi.org/10.4236/ss.2018.96023

Received: April 16, 2018

Accepted: June 26, 2018

Published: June 29, 2018

Copyright ( $) 2018$ by authors and Scientific Research Publishing Inc. This work is licensed under the Creative Commons Attribution-NonCommercial International License (CC BY-NC 4.0).

http://creativecommons.org/licenses/by-nc/4.0/

\begin{abstract}
The cerclage wire is an important adjuvant in the management of Orthopaedic and trauma cases and is particularly useful in long bone fractures. It is especially useful for addressing the fractures, especially the comminuted ones with a butterfly fragment. Orthopaedic cerclage Wiring is used as an adjunct to maintain fracture reduction (either temporarily or permanently), while the bone is primarily stabilised by either a plate intramedullary nail or external fixation frame. Multiple cerclage wires provide adjunctive fixation against compressive shear, bending \& rotational forces and have been used in traumatology since many years. The aim of this article is to introduce a new year simple low cost instrument named as "Suyash cerclage wire passer", with excellent outcomes without any major complications to the Orthopaedic community.
\end{abstract}

\section{Keywords}

Diaphyseal Femur Fracture, Diaphyseal Tibia Fracture, Cerclage Wire, Butterfly Fragment, Comminuted Fractures, Wire Passer, Malleable Metal

\section{Introduction}

The passage of cerclage wire to catch hold of the loose pieces of bone is fraught with dangers to the adjoining neurovascular structures and serious soft tissue insult.

The circumference of the various bones is different. There are various cerclage wire passers available in market. A wire passer, ideally must be able to conform to a variety of bone circumferences. It should be easily able to engage the wire and allow its passage circumferentially around the bone.

However, the commercially available wire passers have a fixed curvature and these instruments often have weak joint where there is a chance of instrument breakage intraoperatively, putting the procedure at risk. The percutaneous cerclage passer device, though useful in many situations, is still quite expensive. 
As the cerclage wire has to negotiate the intermuscular septum, the metallic device should be malleable. By this inherent property of being malleable it should be able to change the curvature or shape while going around the bone as per the bone circumference. Such a malleable soft strong metal i.e. copper is hitherto unused in orthopedics. The blunt tip of the wire passer, with an eye, just pierces and makes a "way" for the eventual passage of the wire.

\section{Procedure}

Under appropriate anesthesia the fracture fragments are exposed and reduced. The butterfly fragment are held securely, in an atraumatic manner, with minimal dissection with the help of bone clamps (Figure 1) A sub-periosteal tunnel is created with a packing tape guaze to push the soft tissue away from the operating field. This ensures the least trauma and insult to the soft tissue around the proposed cerclage wire.

The appropriate length of the cerclage wire is threaded into the eye of the wire passer (Figure 2). The wire passer is then bent and contoured to easily pass around the bone, circumferentially.

Once the tip of wire passer is seen coming out then a gentle, "push rather than a pull" technique, is used to deliver the wire to the other side.

The wire is then knotted in the conventional manner.

\section{Utility of the "Suyash" Cerclage Wire Passer}

The authors have found that this instrument (Figure 3) is useful for all long bones fractures especially in comminuted subtrochanteric femur fractures while performing the cephalo medullary nailing. It is especially useful during arthroplasty if the calcar breaks. It is particularly useful in the tendon fixation to bone using the suture pull out technique. Performing a tension band wire fixation in difficult cases is easy with this instrument.

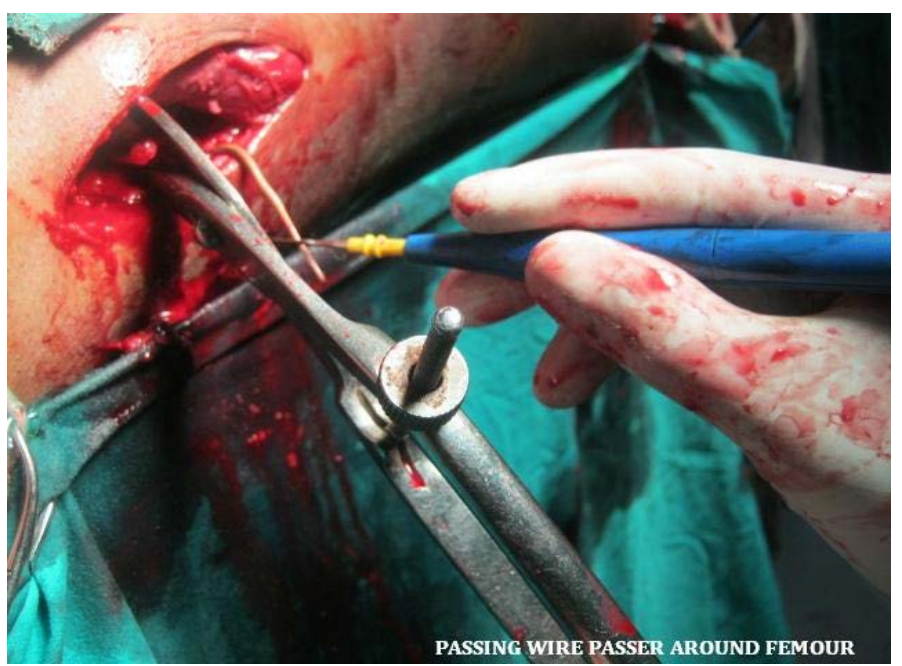

Figure 1. Intraoperative picture with "Suyash" wire passer passed around the bone. 


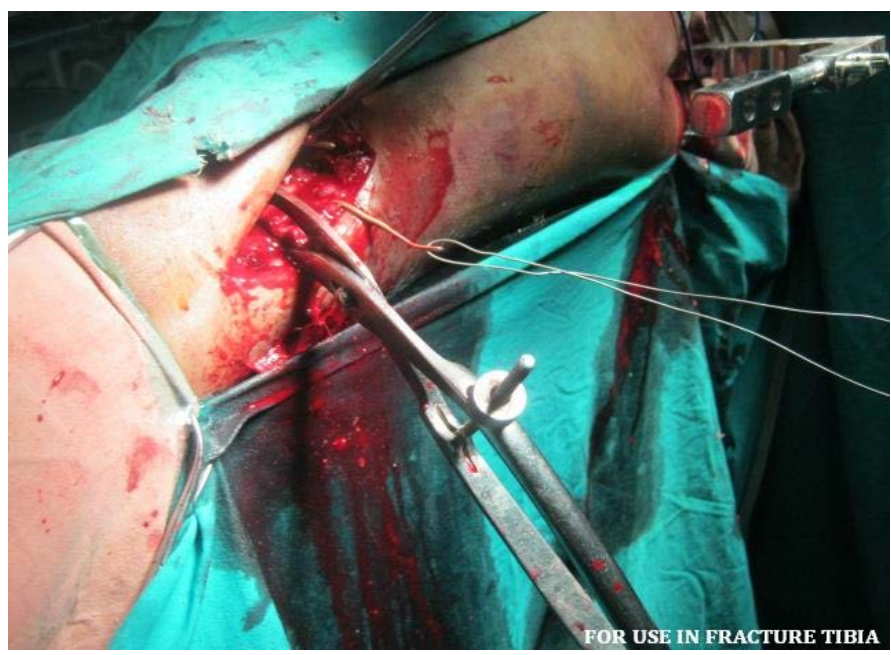

Figure 2. Intraoperative picture with "Suyash" wire passer passed around the bone.

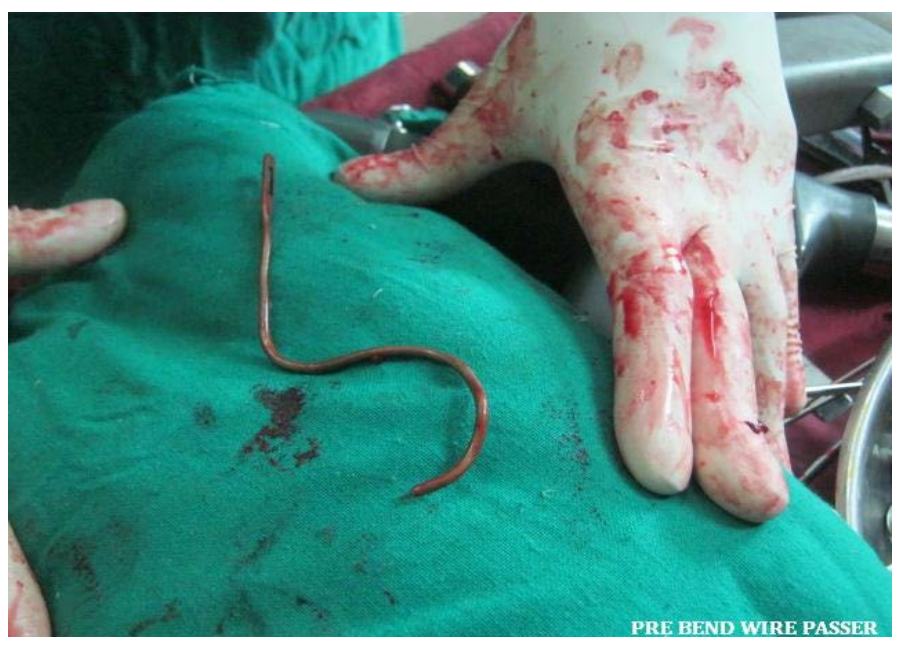

Figure 3. Profile of the "Suyash" wire passer.

\section{Advantages of the "Suyash Cerclage" Wire Passer}

The "Suyash Cerclage" wire passer it was tried on 6 patients of commuted subtrochanteric fracture femur 3 cases of communited fracture Tibia (Figure 4, Figure 5) 2 cases as a tendon passer, once in a wrist tendon injury and another one for ankle injury. One of the most useful advantage, we feel, is it can be locally made and is very cost effective. It can be made under US 5 \$. It decreases the soft tissue dissection and therefore the devitalization. The minimal trauma helps in hastening the union which otherwise cannot be expected with the conventional instruments available in the market. An additional advantage of the wire passer is that you always get a loop which can be divided \& tied at two places.

\section{Discussion}

The cerclage wires have been useful in many situations. Their use in the subtrochanteric and femoral diaphyseal fractures has been mentioned [1]. The results 


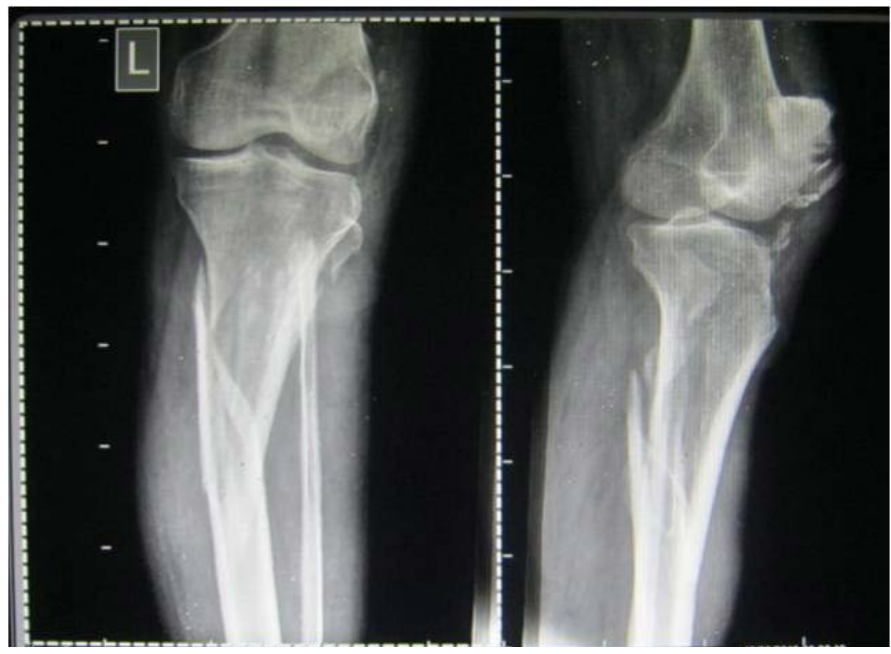

Figure 4. Shows an unstable fracture of the proximal tibia.

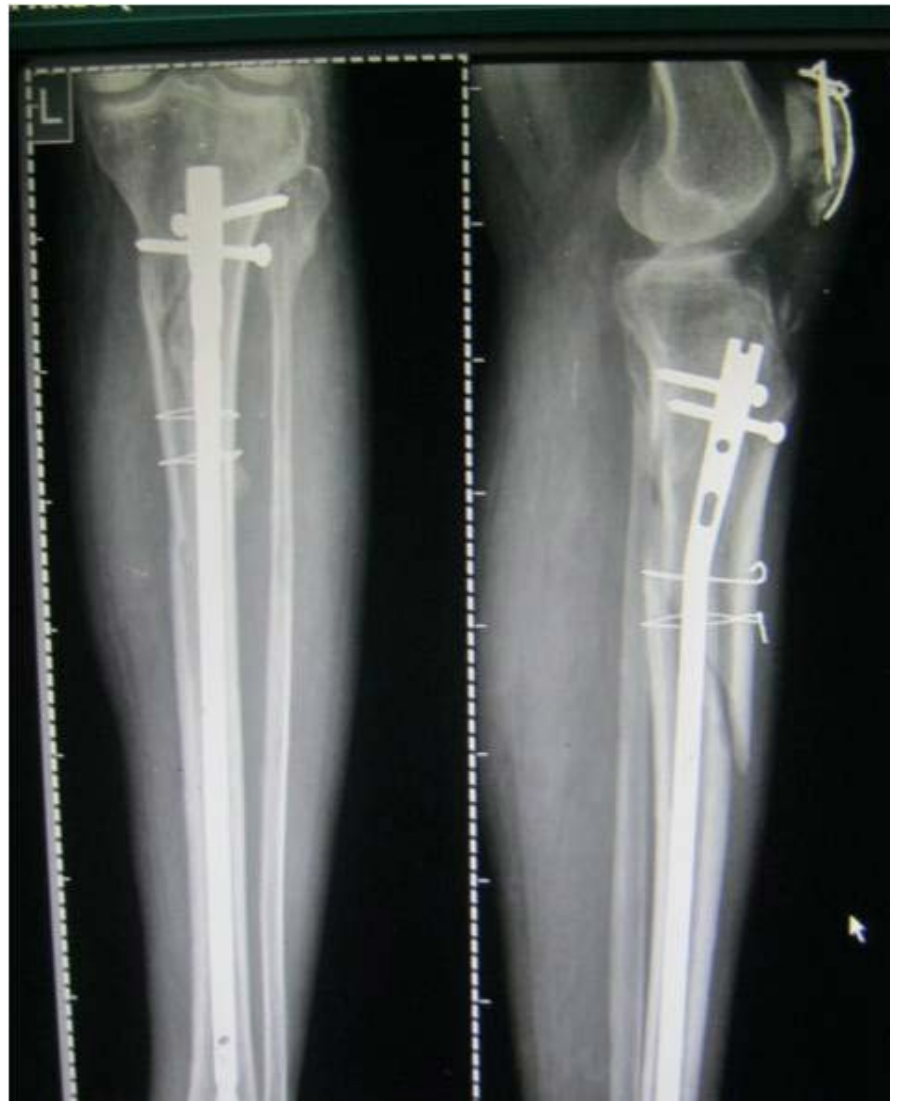

Figure 5. Final fixation with Intramedullary nail in situ provisionally fixed with acerclage wire and IMN.

demonstrate that judicious use of cerclage cables to augment fixation of subtrochanteric femur fractures does not have a deleterious effect on healing unless the number of wires are judiciously used and placed.

Apart from its excellent ability to anatomically reduce the fracture, it increases the overall construct stability and strength, and therefore, increases the load 


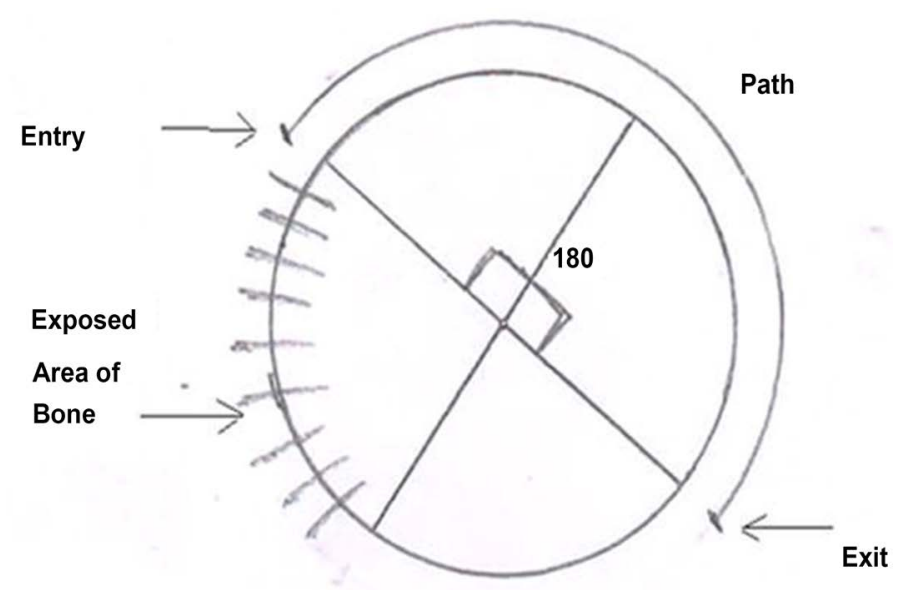

Figure 6. Limited access with the conventional wire passer.

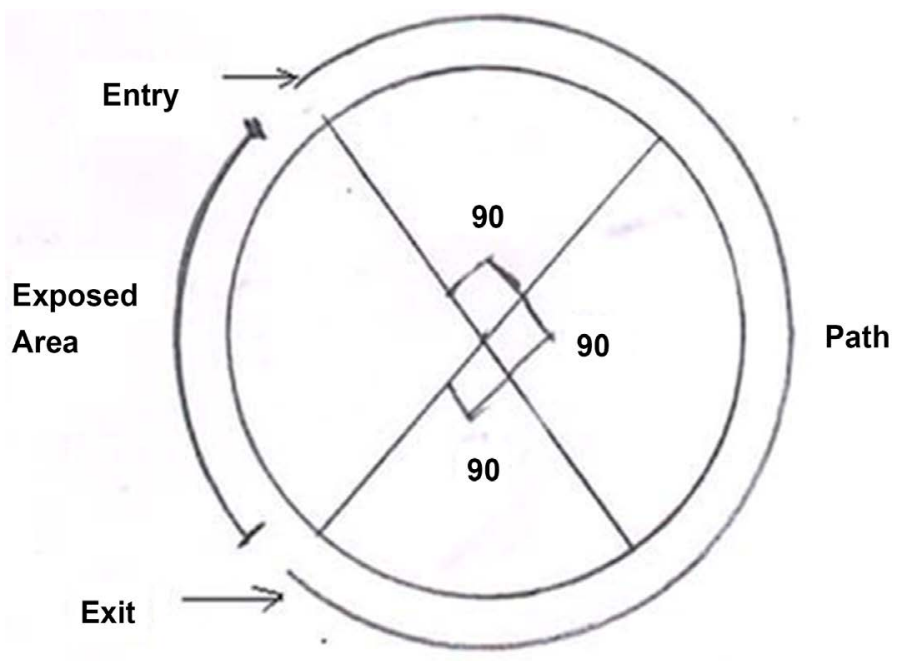

Figure 7. Extended access with the Suyash Universal wire passer.

sharing capacity of the construct, and minimizes fixation failure [2] [3]. Fracture reduction before starting nailing procedure helps substantially to accurately localize the starting point of nail, which is of paramount importance [1] [4].

Major devastating complications such as superficial femoral artery and vein ligation by a femoral mid-shaft cerclage during revision total hip arthroplasty have been reported [5] [6].

It is critical for a surgeon to get a comprehensive pre-operative fracture evaluation and planning, and to set a balance between anatomical and biological fixation to envisage optimal outcome. The judicious usage of the wire passer plays an important role in reducing the soft tissue.

Comparison: advantages.

This instrument can be used for almost all major long bones in the body, multiple uses for a single instrument. The disadvantages which we found out in our short series is that the instrument starts giving way or shows a tendency to break after two or three uses and that is repeated uses may not be possible. 


\section{Concept}

Most of the commercially available cerclage wire passers have a 180 arc of a circle which is very difficult to deliver the cerclage wire without a soft tissue trauma. A 270 arc must deliver the cerclage wire as shown in Figure 6 and Figure 7.

Conclusion: It is a simple instrument, very easy to use and has the potential to revolutionize the use of cerclage wire in Orthopaedic traumatology.

\section{References}

[1] Kim, J.W., Park, K.C., Oh, J.K., Oh, C.W., Yoon, Y.C. and Chang, H.W. (2014) Percutaneous Cerclage Wiring Followed by Intramedullary Nailing for Subtrochanteric Femoral Fractures: A Technical Note with Clinical Results. Arch Orthop Trauma Surg, 134, 1227-1235. https://doi.org/10.1007/s00402-014-2023-7

[2] Ban, I., Birkelund, L. and Palm, H. (2012) Circumferential Wires as a Supplement to Intramedullary Nailing in Unstable Trochanteric Hip Fractures: 4 Reoperations in 60 Patients Followed for 1 Year. Acta Orthopaedica, 83, 240-243. https://doi.org/10.3109/17453674.2012.665329

[3] Finsen, V. (1995) The Effect of Cerclage Wires on the Strength of Diaphyseal Bone. Injury, 26, 159-161. https://doi.org/10.1016/0020-1383(95)93493-2

[4] Tomás, J., Teixidor, J. and Batalla, L. (2013) Subtrochanteric Fractures: Treatment with Cerclage Wire and Long Intramedullary Nail. Journal of Orthopaedic Trauma, 27, e157-e160. https://doi.org/10.1097/BOT.0b013e31826fc03f

[5] Mehta, V. and Finn, H.A. (2005) Femoral Artery and Vein Injury after Cerclage Wiring of the Femur: A Case Report. Journal of Arthroplasty, 20, 811-814. https://doi.org/10.1016/j.arth.2004.12.050

[6] Aleto, T., Ritter, M.A. and Berend, M.E. (2008) Case Report: Superficial Femoral Artery Injury Resulting from Cerclage Wiring during Revision THA. Clinical Orthopaedics and Related Research, 466, 749-753.

https://doi.org/10.1007/s11999-007-0109-Z 